\title{
Rapport annuel national sur la grippe dans le contexte mondial, Canada, 2020-2021
}

\author{
Andrea Nwosu, Liza Lee ${ }^{1}$, Kara Schmidt ${ }^{1}$, Steven Buckrell ${ }^{1}$, Claire Sevenhuysen ${ }^{1}$, Christina Bancej ${ }^{1}$
}

\section{Résumé}

Au cours de la saison de la grippe du Canada en 2020-2021, il n'y a pas eu de circulation communautaire de la grippe. Seulement 69 cas positifs détectés de la grippe ont été signalés, et le pourcentage de réponses positives n'a pas dépassé $0,1 \%$. Les indicateurs de la grippe ont atteint des creux historiques par rapport aux six saisons précédentes, et aucune éclosion de grippe confirmée en laboratoire ni aucun résultat grave n'ont été signalés par les provinces et les territoires. À l'échelle mondiale, la circulation de la grippe était à des niveaux historiquement bas dans les hémisphères du Nord et du Sud. La diminution de l'activité grippale observée au Canada et à l'échelle mondiale coïncide avec la mise en œuvre de mesures de santé publique non pharmaceutiques visant à atténuer la propagation de la maladie à coronavirus 2019 (COVID-19). Bien qu'il soit difficile de prédire quand la grippe commencera à se propager de nouveau, compte tenu de l'augmentation de la vaccination contre la COVID-19 et de l'assouplissement des mesures de santé publique, on peut s'attendre à une résurgence de la grippe qui pourrait être plus grave ou plus intense que les saisons récentes. La vaccination contre la grippe, ainsi que les mesures de santé publique non pharmaceutiques, demeure la meilleure méthode pour prévenir la propagation et l'impact de la grippe. Les autorités de la santé publique doivent demeurer vigilantes, maintenir la surveillance et continuer de planifier la circulation accrue de la grippe saisonnière.
Cette oeuvre est mise à la disposition selon les termes de la licence internationale Creative Commons Attribution 4.0

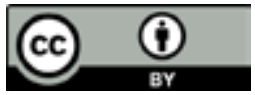

Affiliation

${ }^{1}$ Centre de l'immunisation et des maladies respiratoires infectieuses, Agence de la santé publique du Canada, Ottawa, ON

\section{*Correspondance : \\ fluwatch-epigrippe@phac-aspc. gc.ca}

Citation proposée : Nwosu A, Lee L, Schmidt K, Buckrell S, Sevenhuysen C, Bancej C. Rapport annuel national sur la grippe dans le contexte mondial, Canada, 2020-2021. Relevé des maladies transmissibles au Canada 2021;47(10):450-8. https://doi.org/10.14745/ccdr.v47i10a02f

Mots-clés : grippe, maladie de type grippal, surveillance, état de préparation à la pandémie, éclosions, COVID-19

\section{Introduction}

Les mesures de santé publique mises en œuvre pour réduire la propagation de la maladie à coronavirus 2019 (COVID-19) ont eu une incidence sur la transmission de la grippe dans tous les pays du monde, incluant le Canada (1-4). Au milieu de la saison de la grippe 2020-2021 au Canada (semaine 50, se terminant le 12 décembre 2020), la circulation de la grippe saisonnière au Canada était à des creux sans précédent et n'avait pas atteint le seuil saisonnier (5). Le rapport de surveillance qui suit présente un résumé de la saison annuelle de la grippe de 2020-2021 au Canada (du 23 août 2020 au 28 août 2021) ainsi que des saisons de surveillance de la grippe de l'hémisphère Sud et de I'hémisphère Nord de 2020-2021.

ÉpiGrippe est un programme national de surveillance de la grippe qui surveille la transmission de la grippe et des maladies grippales au Canada. Établi en 1996, il s'agit d'un réseau pancanadien de surveillance de laboratoires, d'hôpitaux, de professionnels de la santé, de Canadiens et de ministères provinciaux et territoriaux de la Santé. Les objectifs de ce programme sont les suivants : 1) déterminer les signaux pour la détection en temps opportun des épidémies et d'autres événements préoccupants sur le plan de la santé publique, et coordonner l'évaluation et l'intervention; 2) contribuer à la base de données probantes nécessaire à la planification; l'élaboration et la mise en œuvre de programmes de santé et de politiques publiques saines pour le contrôle de la grippe; et 3) mise en place d'une solide infrastructure de surveillance pour l'intervention et la recherche opportunes et pertinentes nécessaires pour atténuer les répercussions de la grippe (6).

\section{Méthode}

\section{Conception}

Le programme Surveillance ÉpiGrippe effectue une surveillance prospective de la grippe et du syndrome grippal. Chaque année, la surveillance de la grippe a lieu de la semaine épidémiologique 35 à la semaine 34 de l'année suivante. Le programme Surveillance ÉpiGrippe est un système de surveillance composite comprenant la surveillance virologique, 
la surveillance syndromique, la surveillance de l'activité grippale, la surveillance des éclosions, la surveillance des conséquences graves et la surveillance des vaccins.

\section{Définitions des indicateurs}

Les définitions normalisées des indicateurs de base qui sont surveillés dans le cadre du programme Surveillance ÉpiGrippe et qui sont présentées dans le présent rapport sont définies au tableau 1.

\section{Tableau 1 : Indicateurs de base de Surveillance ÉpiGrippe utilisés pour résumer la saison de la grippe 2020-2021 au Canada}

Indicateur

Taux de positivité à la grippe

Caractérisation de la

souche grippale
Proportion hebdomadaire de tests de diagnostic positifs pour la grippe par rapport à tous les tests de diagnostic effectués.

Le nombre d'isolats de grippe caractérisés par le Laboratoire national de microbiologie comparativement aux isolats récents canadiens et mondiaux et aux souches de virus de vaccin recommandées par l'Organisation mondiale de la Santé.

\section{Proportion de visites La proportion hebdomadaire de visites de} pour le syndrome grippal patients à des praticiens sentinelles en raison d'un syndrome grippal par rapport à toutes les visites de patients.

La proportion hebdomadaire de participants qui déclarent eux-mêmes la toux et la fièvre par rapport à tous les participants.

participants au programme

ActionGrippe avec un syndrome grippal Éclosions de grippe

\section{Hospitalisations}

associées à la grippe
Nombre d'éclosions de grippe ou de syndrome grippal par établissement et type de grippe.

Le nombre et le taux d'hospitalisations associées à la grippe.

\section{Calcul}

Numérateur : Nombre de détections de la grippe.

Dénominateur : Nombre total de tests diagnostiques de la grippe traités.

Comptages et proportions semblables/ différentes sur le plan antigénique par rapport aux souches du vaccin.

Numérateur: Nombre de visites de patients pour un syndrome grippal observées aux sites de sentinelles.

Dénominateur : Nombre total de patients vus aux sites de sentinelles, quelle qu'en soit la raison.

Numérateur : Nombre de participants qui déclarent avoir de la toux et de la fièvre.

Dénominateur : Nombre total de participants faisant des rapports.

Dénombrements et proportions des éclosions selon l'établissement et le type de grippe/ syndrome grippal.

Chiffres et taux pour 100000 habitants.

\section{Source des données}

Système de surveillance de la détection de virus respiratoires et FluNet

Laboratoire national de

microbiologie

Système de rapports du syndrome grippal par les praticiens sentinelles

Participants au programme ActionGrippe

Autorités provinciales et territoriales en matière de santé publique

Autorités provinciales et territoriales de la santé publique, Programme de surveillance active des effets secondaires associés aux vaccins Réseau canadien des registres d'immunisation.

La réduction proportionnelle de la grippe chez les personnes vaccinées, par rapport à celles qui ne sont pas vaccinées parmi le syndrome grippal médicalement confirmé.
Les estimations de l'efficacité du vaccin sont fondées sur les résultats d'une étude de cas témoin modifiée (essai de conception négatif) et calculées à l'aide de l'équation suivante :

$$
V E=100 \% *\left(1-\frac{o_{\text {pas }}}{o_{\text {oug }}}\right)
$$

où $\mathrm{O}_{\text {pos }}$ est la probabilité de vaccination parmi les personnes testées positives pour la grippe et $\mathrm{O}_{\text {neg }}$ est la probabilité de vaccination parmi les personnes testées négatives.

Les estimations de l'efficacité du vaccin sont fondées sur les résultats d'une étude de cas témoin modifiée (essai de conception négatif) et calculées à l'aide de l'équation suivante :

$V E=100 \% *\left(1-\frac{o_{p o s}}{O_{\text {neg }}}\right)$

où $\mathrm{O}_{\text {pos }}$ est la probabilité de vaccination parmi les personnes testées positives pour la grippe et $\mathrm{O}_{\text {ne }}$ est la probabilité de vaccination parmi les personnes testées négatives.

Couverture de la vaccination contre la grippe
Pourcentage de Canadiens âgés de six mois et plus qui ont reçu une dose du vaccin contre la grippe saisonnière pendant la saison grippale en cours.
Numérateur : Nombre de personnes qui ont reçu le vaccin contre la grippe pendant cette saison.

Dénominateur: Nombre de personnes admissibles au vaccin cette saison-là.
Réseau canadien de surveillance des praticiens sentinelles

Réseau canadien des registres d'immunisation

nquête nationale sur la couverture vaccinale grippale de l'Agence de la santé publique du Canada 


\section{Source de données}

Données virologiques canadiennes : Des données agrégées et au niveau des cas sur la détection de la grippe sont transmises à ÉpiGrippe par l'entremise du programme de surveillance et de détection de virus des voies respiratoires (7). Le programme de surveillance et de détection de virus des voies respiratoires est un système sentinelle en laboratoire qui surveille la circulation temporelle des virus respiratoires au Canada à l'échelle nationale et régionale. Ce système de surveillance consiste en 34 laboratoires qui déclarent le nombre d'analyses effectuées et le nombre de spécimens positifs pour la grippe et d'autres virus respiratoires. Les spécimens de chaque province et territoire sont représentés dans les données virologiques. Les laboratoires provinciaux fournissent des données individuelles au niveau des cas.

Données de caractérisation génétique et antigénique et données de susceptibilité antivirale : Une proportion des détections de la grippe confirmées en laboratoire sont soumises à des tests de caractérisation génétique et antigénique et de susceptibilité antivirale. Les résultats sont fournis par le Laboratoire national de microbiologie de l'Agence de la santé publique du Canada.

Données virologiques mondiales : FluNet est un outil Web mondial de surveillance virologique de la grippe (8). Les données virologiques des centres et laboratoires nationaux de lutte contre la grippe dans 75 pays sont transmises par cette plateforme. Des données agrégées sur les tests de dépistage et les détections de la grippe provenant de pays et de continents du Nord (États-Unis, Europe) et de l'hémisphère Sud (Australie, Chili, Afrique du Sud) ont été extraites de la plateforme FluNet de l'Organisation mondiale de la Santé le 11 septembre 2021. Les chiffres mis à jour des détections du Chili n'étaient pas disponibles dans l'extrait de la base de données FluNet du 11 septembre 2021; par conséquent, une extraction de données antérieure du 27 juillet 2021 a été utilisée.

Données sur les niveaux d'activité et les éclosions : Tous les services de santé publique provinciaux et territoriaux fournissent une évaluation catégorique (aucune activité, sporadique, localisée ou répandue) de l'intensité et de la propagation géographique de la grippe (niveau d'activité) ainsi que le nombre d'éclosions de grippe et de syndrome grippal selon l'établissement, le type et le sous-type des régions de surveillance dans leur administration.

Données syndromiques : Les données syndromiques proviennent de deux systèmes : le système de surveillance sentinelle du syndrome grippal et le programme ActionGrippe. Le système de surveillance sentinelle du syndrome grippal est constitué de professionnels de la santé de partout au Canada qui déclarent le nombre de patients ayant subi un syndrome grippal et le nombre total de patients vus par groupe d'âge.
Les participants au programme ActionGrippe sont composés de volontaires de partout au Canada qui déclarent chaque semaine, au moyen d'un questionnaire en ligne, s'ils ont eu des symptômes de type grippal.

Données de surveillance des cas graves : Les données sur les cas graves associés à la grippe (i.e. hospitalisation, admissions et décès aux soins intensifs) proviennent de trois sources : 1) ministères provinciaux et territoriaux de la Santé surveillance des cas graves; 2 ) Programme de surveillance active des effets secondaires associés aux vaccins; et 3 ) le réseau de surveillance des cas graves du Réseau canadien de recherche sur l'immunisation.

Surveillance des cas graves dans les provinces et les territoires : Neuf ministères provinciaux et territoriaux de la Santé du Canada (Alberta, Manitoba, Saskatchewan, Nouvelle-Écosse, Nouveau-Brunswick, Terre-Neuve-et-Labrador, Île-du-Prince-Édouard, Yukon et Territoires du Nord-Ouest) participent et communiquent des renseignements sur les cas (âge, type/sous-type de grippe associé) pour les hospitalisations, les admissions aux soins intensifs et les décès associés à la grippe.

Données sur les cas graves associés à la grippe chez les enfants (16 ans et moins) : Le Programme de surveillance active des effets secondaires associés aux vaccins est un réseau sentinelle d'hôpitaux pédiatriques qui comprend 12 hôpitaux pédiatriques répartis dans huit provinces du Canada. Des renseignements détaillés au niveau des cas, comme l'âge, le type et le sous-type de grippe, le sexe, les problèmes médicaux sous-jacents, l'état de vaccination et le traitement des hospitalisations associées à la grippe, les admissions aux soins intensifs et les décès chez les patients, sont déclarés chaque semaine.

Données sur les cas graves associés à la grippe chez les adultes (16 ans et plus) : Le Réseau canadien de recherche sur l'immunisation est un réseau sentinelle d'hôpitaux pour adultes qui comprend dix hôpitaux répartis dans quatre provinces. Ce réseau fournit des renseignements détaillés au niveau des cas pour les hospitalisations associées à la grippe, l'admission aux soins intensifs et les décès de patients hospitalisés.

Données sur la surveillance des vaccins : Les données sur la couverture des vaccins contre la grippe sont fournies par l'Enquête nationale sur la couverture vaccinale grippale de l'Agence de la santé publique du Canada. Les données sur l'efficacité du vaccin sont fournies par deux réseaux, le Réseau canadien de surveillance des praticiens sentinelles et le réseau de surveillance des cas graves du Réseau canadien de recherche sur l'immunisation. Le Réseau canadien de surveillance des praticiens sentinelles fournit des estimations de la façon dont le vaccin contre la grippe prévient les visites en soins primaires pour la 
de virus grippaux confirmées en laboratoire ont été signalées pendant la saison de la grippe 2020-2021, toutes représentant une activité sporadique; 31 de ces détections ont été associées à la réception d'un vaccin antigrippal vivant atténué $(9,10)$. La grippe de type A représentait $67 \%(n=46)$ des détections déclarées. Seuls 20 virus de la grippe A ont été sous-typés; par conséquent, les caractéristiques des sous-types des détections sporadiques n'ont pu être déterminées.

Malgré le peu de détections signalées, des niveaux élevés de tests de dépistage de la grippe supérieurs aux moyennes saisonnières ont été maintenus tout au long de la saison de la grippe 2020-2021, avec un total de 632580 tests signalés. Par le passé, pendant les saisons de surveillance hors du contexte de la pandémie de 2014-2015 à 2018-2019, le nombre total de tests effectués variait de 237777 à 391 862. Le pourcentage de tests de laboratoire positifs pour la grippe est demeuré exceptionnellement bas tout au long de la saison de la grippe 2020-2021. Le pourcentage de positivité déclaré a varié de $0,0 \%$ à $0,1 \%$ cette saison comparativement à une moyenne historique de $0,8 \%$ à $25,1 \%$, tandis que les tests tout au long de la saison ont été à peu près deux fois plus élevés que la moyenne historique (figure 1).

Il n'y a pas eu de circulation communautaire de la grippe au Canada pendant la saison 2020-2021. Au total, 69 détections

Figure 1 : Nombre de tests de dépistage de la grippe et pourcentage de tests positifs, au Canada, aux États-Unis et en Europe, selon la semaine de surveillance

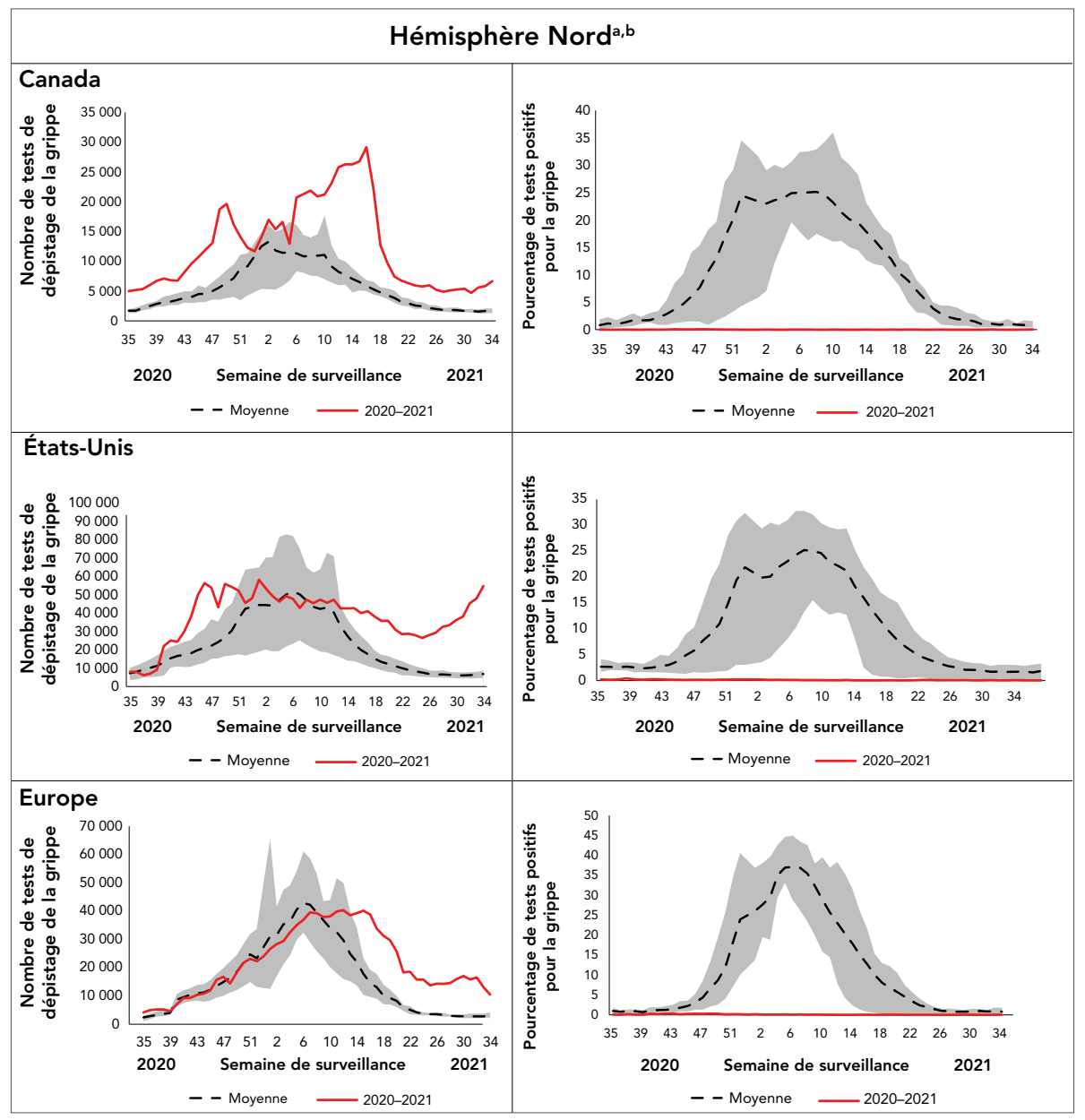


Figure 1 : Nombre de tests de dépistage de la grippe et pourcentage de tests positifs, au Canada, aux États-Unis et en Europe, selon la semaine de surveillance (suite)

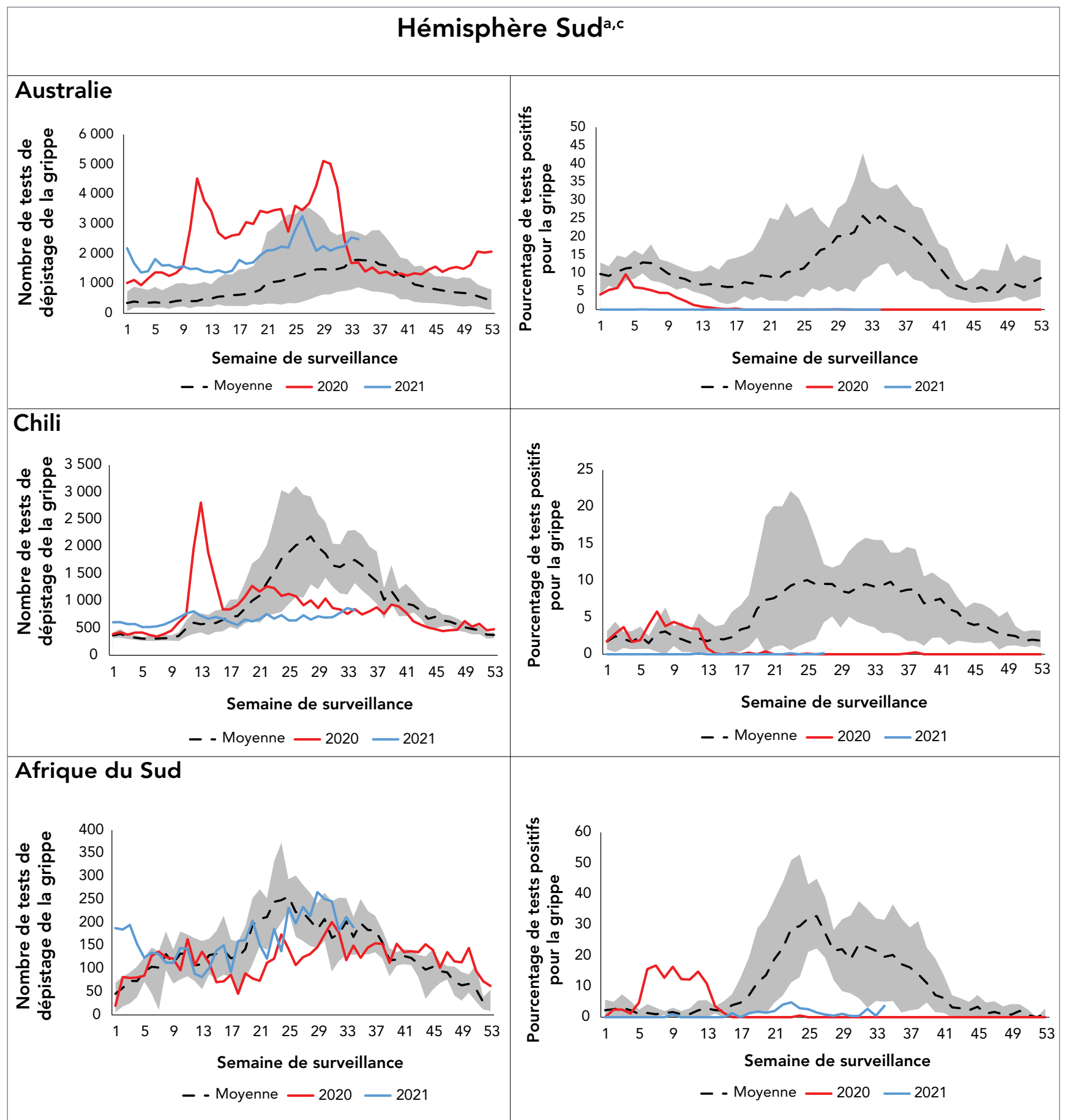

a La zone ombrée représente le nombre maximal et minimal de tests effectués (à gauche), le pourcentage de tests de dépistage de la grippe positifs (à droite), par semaine de surveillance, des

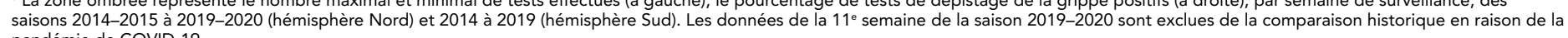
pandémie de COVID-19

b Saison de la grippe de l'hémisphère Nord 2020-2021 au Canada, aux États-Unis et en Europe comparativement à la moyenne historique (saisons 2014-2015 à 2019-2020)

' Saisons de la grippe de l'hémisphère Sud 2020-2021 en Australie, au Chili et en Afrique du Sud comparativement à la moyenne historique (saisons 2014 à 2019) 
À l'échelle internationale, on a observé une tendance semblable de diminution de la circulation de la grippe pendant les saisons de la grippe de l'hémisphère Sud de 2020, de l'hémisphère Nord de 2020-2021 et de l'hémisphère Sud de 2021 (figure 1). Dans I'hémisphère Sud, l'activité grippale s'est produite au cours de la première partie de la saison de surveillance de 2020, puis a diminué et est demeurée à des niveaux exceptionnellement bas à la semaine 14 de 2020. Le pourcentage déclaré de positivité en Australie (de $0,0 \%$ à 9,7 \%), au Chili (de $0,0 \%$ à $5,8 \%$ ) et en Afrique du Sud (de $0,0 \%$ à 16,8 \%) était inférieur à leur fourchette historique respective de $4,8 \%$ à $25,7 \%$, de $1,5 \%$ à $10,1 \%$ et de $0,0 \%$ à $32,8 \%$. De même, aux États-Unis et en Europe, le pourcentage de tests de laboratoire positifs pour la grippe est demeuré exceptionnellement bas (figure 1). Au cours de la saison 2020-2021, le pourcentage de positivité aux États-Unis et en Europe n'a pas dépassé $0,4 \%$ et $0,4 \%$, respectivement. Alors que la positivité moyenne historique variait de $1,6 \%$ à $25,2 \%$ et de $0,7 \%$ à $37,1 \%$. En date de la semaine 34 (semaine 27 pour le Chili), l'activité grippale est demeurée à des niveaux exceptionnellement bas en 2021 dans I'hémisphère Sud, le taux de positivité de la grippe ne dépassant pas 3,9\% en Australie, au Chili et en Afrique du Sud (figure 1).

\section{Caractérisation de la souche et tests de résistance antivirale}

En raison de la faible circulation de la grippe au Canada, seulement cinq virus de la grippe ont été caractérisés cette saison, ce qui était considérablement inférieur à ce qui est typiquement caractérisé par le Laboratoire national de microbiologie pendant une saison de surveillance de la grippe ( $n=1171$ à 3857 de 2014-2015 à 2019-2020). Parmi ces cinq virus, l'un était un virus de la grippe saisonnière $B$ et les quatre autres étaient des infections zoonotiques. Tous les virus soumis provenaient de l'Alberta et du Manitoba.
Le virus de la grippe $B$ caractérisé était antigéniquement lié à la souche B/Washington/02/2019 (Victoria). Les quatre infections zoonotiques étaient les variants H1N2v $(n=2), H 3 N 2 v(n=1)$ et H1N1v $(n=1)(11-14)$.

\section{Syndromique}

Les deux programmes de surveillance syndromique d'ÉpiGrippe ont montré une activité plus faible que la normale, ce qui n'est pas inattendu étant donné l'absence de circulation communautaire de la grippe. Tout au long de la saison, il y a eu de petites fluctuations des activités de syndrome grippal (figure 2). Ces fluctuations de l'activité étaient probablement des signaux d'une autre activité virale respiratoire comme l'entérovirus, le rhinovirus, le virus respiratoire syncytial, I'adénovirus et le coronavirus du syndrome respiratoire aigu sévère 2 (SRAS-CoV-2).

\section{Système de surveillance du syndrome grippal des praticiens sentinelles}

Le système de surveillance du syndrome grippal des praticiens sentinelles a rapporté des pourcentages inférieurs à la moyenne des consultations attribuables au syndrome grippal comparativement aux saisons précédentes. Les pourcentages hebdomadaires de consultations attribuables au syndrome grippal ont varié de $0,06 \%$ à $0,49 \%$ (comparativement à la moyenne sur six ans de $0,41 \%$ à 3,32 \%). Cela n'est pas inattendu compte tenu des changements dans le comportement de recherche de soins, des options de soins supplémentaires pour les personnes présentant des symptômes de syndrome grippal, d'une réduction du nombre de signalements de sentinelles et du nombre moyen de patients examinés chaque semaine. Au cours de la saison précédente, une moyenne hebdomadaire de 94 sentinelles ont fait des déclarations et une moyenne de 8775 patients ont été examinés, comparativement

Figure 2 : Pourcentage de consultations pour des maladies de type grippal signalées par les praticiens du système de surveillance sentinelle du syndrome grippal et les praticiens sentinelles participants pour la fièvre et la toux ${ }^{a}$, par semaine de surveillance au Canada, saison 2020-2021, comparativement à la moyenne historique

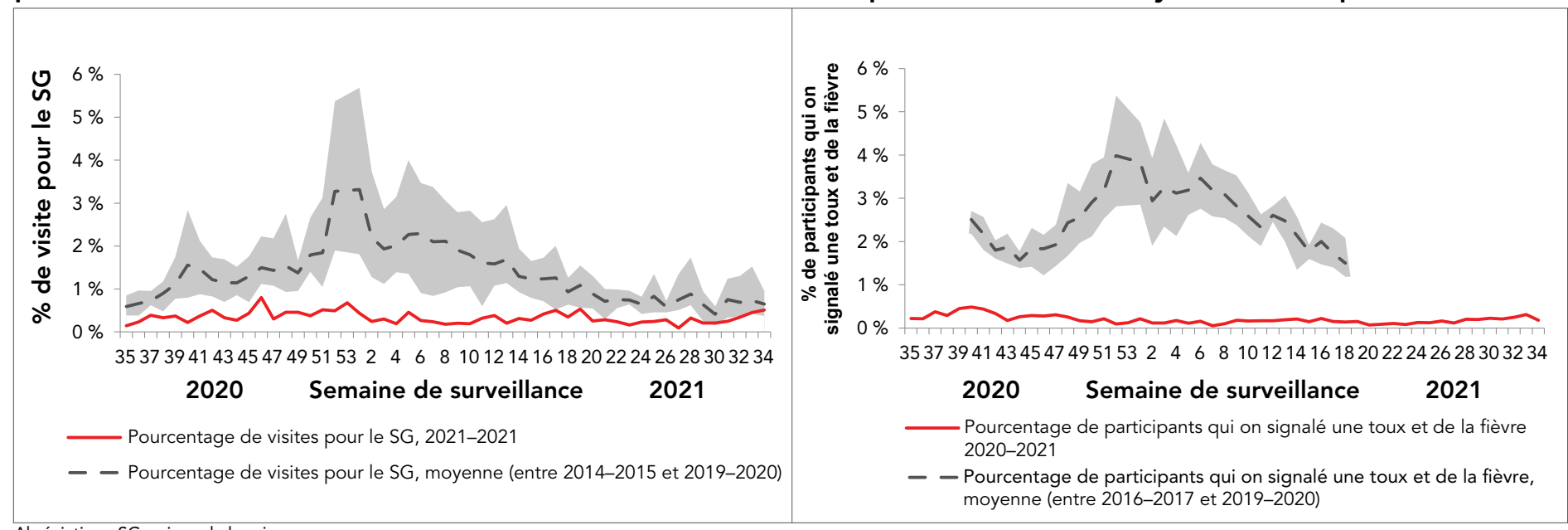

Abréviation : SG, saison de la grippe

La zone ombrée représente le pourcentage maximal et minimal de consultations

(à droite) par semaine de surveillance pour les saisons 2014-2015 à 2019-2020

Remarque : Les données de la $11^{\mathrm{e}}$ semaine de la saison 2019-2020 sont exclues de la comparaison historique en raison de la pandémie de COVID-19 
à la moyenne hebdomadaire de 62 déclarations de sentinelles pour la saison en cours et à une moyenne de 5503 patients examinés.

Pendant la majeure partie de la saison, le pourcentage hebdomadaire le plus élevé de visites liées au syndrome grippal a été déclaré chez les personnes de moins de 20 ans. Le plus faible pourcentage de visites liées au syndrome grippal a été déclaré chez les adultes de 65 ans et plus. Cette tendance était semblable à celle observée au cours des saisons précédentes, malgré le nombre réduit de patients et les pourcentages plus faibles de visites attribuables au syndrome grippal.

\section{ActionGrippe}

Les participants au programme ActionGrippe ont signalé des pourcentages inférieurs à la moyenne de participants ayant déclaré avoir de la fièvre et de la toux par rapport aux saisons précédentes. Le pourcentage hebdomadaire de rapports de fièvre et de toux a varié de $0,1 \%$ à $0,5 \%$, comparativement à la moyenne sur quatre ans de $1,5 \%$ à $4,0 \%$. En moyenne, 12048 participants se sont présentés chaque semaine (plage de 9290 à 12 831), soit environ 3,5 fois plus que la saison précédente. Les signalements du programme ActionGrippe ne sont pas affectés par les changements dans les services de santé ou les comportements de recherche de la santé; toutefois, ces faibles signalements de toux et de fièvre peuvent être un effet direct des mesures de santé individuelles ou publiques adoptées pour réduire la propagation de la COVID-19.

Comme dans le cas de la surveillance sentinelle syndromique par les praticiens de la santé, pendant la majeure partie de la saison, les pourcentages hebdomadaires les plus élevés de participants ayant déclaré tousser et faire de la fièvre étaient chez les jeunes de moins de 20 ans. Les pourcentages hebdomadaires les plus faibles de participants déclarant tousser et faire de la fièvre étaient chez les adultes de 65 ans et plus. Cette tendance est semblable à celle des saisons précédentes.

\section{Éclosions}

Toutes les éclosions signalées pendant la saison $(n=138)$ étaient des éclosions de syndrome grippal dans les écoles et les garderies. Le nombre d'éclosions de syndrome grippal dans les écoles et les garderies était plus élevé comparativement aux deux saisons précédentes. Cela n'est pas inattendu compte tenu des changements apportés à la surveillance des éclosions, en particulier la vigilance accrue dans les écoles pour surveiller et signaler l'absentéisme en raison du syndrome grippal, et les restrictions accrues sur la présence des enfants qui présentent des symptômes de maladie respiratoire virale.

Aucune éclosion de grippe confirmée en laboratoire n'a été signalée cette saison.

\section{Cas graves}

Aucune hospitalisation liée à la grippe n'a été déclarée par les provinces et les territoires participants (Alberta, Manitoba, Nouveau-Brunswick, Terre-Neuve-et-Labrador, Territoires du Nord-Ouest, Nouvelle-Écosse, île-du-Prince-Édouard et Yukon).

Aucune hospitalisation liée à la grippe n'a été signalée par le réseau sentinelle d'hospitalisation pour adultes (Réseau canadien de recherche sur l'immunisation) et moins de cinq hospitalisations liées à la grippe ont été signalées par le réseau sentinelle d'hospitalisation pour enfants (Programme de surveillance active des effets secondaires associés aux vaccins).

\section{Surveillance des vaccins}

L'Organisation mondiale de la Santé a recommandé que le vaccin contre la grippe à base d'œufs de l'hémisphère Nord de 2020-2021 contienne les souches suivantes (15) :

- Virus de type A/Guangdong-Maonan/SWL1536/2019 (H1N1)pdm09

- $\quad$ Virus de type A/Hong Kong/2671/2019 (H3N2)

- Virus de type B/Washington/02/2019 (lignée B/Victoria)

- Virus de type B/Phuket/3073/2013 (lignée B/Yamagata) (vaccin quadrivalent uniquement)

\section{Couverture vaccinale}

La couverture vaccinale pour la saison de la grippe 2020-2021 était semblable à celle de la saison précédente. Trente-deux pour cent des adultes de 18 à 64 ans ont reçu leur vaccin antigrippal (10). La couverture vaccinale était plus élevée chez les personnes âgées de 65 ans et plus (70\%) et les adultes ayant des problèmes médicaux chroniques (40\%). La couverture vaccinale globale était plus élevée chez les femmes que chez les hommes.

\section{Efficacité du vaccin}

En raison de l'absence de diffusion saisonnière de la grippe, aucune estimation de l'efficacité du vaccin contre la grippe n'a pu être produite pour le Canada ni pour aucun pays de I'hémisphère Nord ou Sud depuis la saison de la grippe de I'hémisphère Nord 2019-2020.

\section{Discussion}

La saison canadienne de la grippe 2020-2021 a été caractérisée par une détection sporadique de la grippe et l'absence d'une circulation soutenue du virus dans la collectivité. Au Canada, des mesures de santé publique non pharmaceutiques (i.e. fermetures d'écoles, restrictions de déplacement, masque obligatoire et augmentation du lavage des mains) ont été mises en œuvre en mars 2020 et maintenues tout au long de la saison de la grippe 2020-2021 afin de réduire la transmission de la COVID-19. L'analyse épidémiologique des données de laboratoire sur la grippe a démontré que ces mesures étaient efficaces pour réduire l'incidence et l'impact de la grippe au Canada $(3,4)$. Malgré des tests de dépistage de virus 
respiratoires élevés cette saison, seulement 69 virus de la grippe ont été détectés, et le pourcentage de positivité à la grippe n'a pas dépassé 0,1\%. Historiquement, en moyenne, 52169 virus de la grippe ont été détectés au cours d'une saison, le pourcentage de positivité variant de $0,8 \%$ à $25,1 \%$. On a également observé une diminution de la circulation grippale dans d'autres pays du monde. Les données de laboratoire soumises au programme FluNet de l'Organisation mondiale de la Santé ont montré que pendant les saisons de la grippe de I'hémisphère Sud de 2020, de l'hémisphère Nord de 2020-2021 et de l'hémisphère Sud de 2021, l'Australie, le Chili, l'Afrique du Sud, les États-Unis et les pays d'Europe ont également connu une diminution de l'activité grippale pendant la majeure partie de leur saison de surveillance. En date de la semaine 34 de 2021 (semaine 27 pour le Chili), les données de laboratoire de la saison de la grippe de l'hémisphère Sud de 2021 ont montré que l'activité grippale est demeurée faible, car les mesures de santé publique visant à atténuer la transmission de la COVID-19 sont demeurées en place ou ont été rétablies lorsque les cas de COVID-19 ont augmenté.

L'absence d'épidémies de grippe a des répercussions qui nécessiteront une vigilance accrue. En raison de la diminution de la circulation de la grippe au cours des deux saisons précédentes, un faible niveau d'immunité au sein de la population, en particulier chez les nourrissons et les enfants plus jeunes, pourrait entraîner des taux d'infection plus élevés une fois que la circulation de la grippe sera rétablie à des niveaux réguliers $(15,16)$. De plus, compte tenu de la diminution de la circulation de la grippe, il pourrait y avoir une possibilité accrue de non-concordance entre les vaccins contre la grippe (15). Les recommandations sur la composition des virus du vaccin contre la grippe chaque année sont faites en prédisant quels virus circuleront au cours de la prochaine saison de surveillance (17). Ces prévisions sont fondées sur des données de surveillance en laboratoire qui indiquent quels virus circulent actuellement (17). Compte tenu de la rareté des données de surveillance en raison de la réduction de la surveillance et de la circulation de la grippe, la capacité de prédire avec précision quels virus circuleront peut être réduite (15). Par ailleurs, à mesure que la couverture vaccinale contre la COVID-19 augmente et que les pays commencent à assouplir les mesures de santé publique, des souches grippales semblables à celles des années avant la pandémie ou de nouvelles souches peuvent apparaître, entraînant des épidémies de grippe plus importantes que la normale (15). Bien qu'il soit actuellement difficile de prédire si la circulation de la grippe demeurera faible au Canada et dans le monde pour la saison de la grippe 2021-2022, les autorités de la santé publique doivent demeurer vigilantes et continuer de planifier la circulation saisonnière de la grippe et maintenir la capacité de diagnostic et de surveillance des laboratoires. La vaccination contre la grippe et les mesures de santé publique non pharmaceutiques demeurent les meilleures méthodes pour prévenir la propagation et l'impact de la grippe, surtout compte tenu de l'incertitude quant à la gravité et à l'intensité de la circulation des virus respiratoires au cours des prochaines saisons.

\section{Déclaration des auteurs}

L'équipe de Surveillance ÉpiGrippe du Centre de l'immunisation et des maladies respiratoires infectieuses a élaboré la première ébauche en collaboration; tous les auteurs ont contribué à la conceptualisation, à la rédaction et à la révision de l'article.

\section{Intérêts concurrents}

Aucun.

\section{Remerciements}

Merci beaucoup à tous ceux qui, partout au Canada, contribuent à la surveillance de la grippe. Le programme Surveillance ÉpiGrippe comprend un réseau bénévole de laboratoires, d'hôpitaux, de cabinets de médecins, de ministères provinciaux et territoriaux de la Santé et de Canadiens qui contribuent au programme ActionGrippe. Nous reconnaissons également la contribution des réseaux de surveillance et de recherche suivants qui contribuent à l'amélioration de la surveillance et de l'échange de connaissances sur l'efficacité du vaccin contre la grippe pour ÉpiGrippe : le Programme de surveillance active des effets secondaires associés aux vaccins, le réseau de surveillance des cas graves du Réseau canadien de recherche sur l'immunisation, le Réseau de surveillance des résultats graves et le Réseau canadien de surveillance des praticiens sentinelles de la grippe. Enfin, nous tenons à remercier la Section de la caractérisation des souches et des tests de résistance antivirale du Laboratoire national de microbiologie pour la caractérisation des virus grippaux et respiratoires et la Section de la couverture vaccinale du Centre de l'immunisation et des maladies respiratoires infectieuses pour leur analyse des enquêtes nationales annuelles sur la couverture vaccinale saisonnière.

\section{Financement}

Le programme Surveillance ÉpiGrippe est financé par l'Agence de la santé publique du Canada.

\section{Références}

1. Olsen SJ, Azziz-Baumgartner E, Budd AP, Brammer L, Sullivan S, Pineda RF, Cohen C, Fry AM. Decreased Influenza Activity During the COVID-19 Pandemic - United States, Australia, Chile, and South Africa, 2020. MMWR Morb Mort Wkly Rep. 2020;69:1305-9. https://www.cdc.gov/mmwr/ volumes/69/wr/mm6937a6.htm 
2. World Health Organization. Global Influenza Programme. Influenza Update No 398. Geneva, Switzerland: WHO; 2021 (accédé 2021-07-28). https://cdn.who. int/media/docs/default-source/influenza/influenzaupdates/2021/2021_07_19_surveillance_update_398.pdf?sfv rsn=cc48538f_5\&download=true

3. Groves HE, Piché-Renaud PP, Peci A, Farrar DS, Buckrell S, Bancej C, Sevenhuysen C, Campigotto A, Gubbay JB, Morris SK. The impact of the COVID-19 pandemic on influenza, respiratory syncytial virus, and other seasonal respiratory virus circulation in Canada: A population-based study. Lancet Reg Health Am 2021;1:100015. DOI PubMed

4. Lagacé-Wiens P, Sevenhuysen C, Lee L, Nwosu A, Smith T. Incidence des interventions non pharmaceutiques sur la détection de l'influenza $A$ et $B$ en laboratoire au Canada. Relevé des maladiestransmissibles au Canada 2021;47(3):155-61. DOI

5. Lee L, Butt K, Buckrell S, Nwosu A, Sevenhuysen C, Bancej C. Rapport national de mi-saison sur l'influenza au Canada, 2020-2021. Relevé des maladies transmissibles au Canada 2021;47(1):1-5. DOI

6. Agence de la santé publique du Canada. Grippe (influenza) : Surveillance. Vue d'ensemble de la surveillance de la grippe au Canada. Ottawa (ON) : ASPC; 2021 (accédé 2021-07-28). https://www.canada.ca/fr/sante-publique/services/maladies/ grippe-influenza/surveillance-influenza/propos-surveillanceinfluenza.html

7. Agence de la santé publique du Canada. Grippe (influenza) : Surveillance. Détection de virus des voies respiratoires au Canada. Ottawa (ON) : ASPC; 2021 (accédé 2021-07-28). https://www.canada.ca/fr/sante-publique/services/ surveillance/detection-virus-voies-respiratoires-canada.html

8. World Health Organization. Global Influenza Programme. FluNet. Geneva, Switzerland: WHO; 2021 (accédé 2021-07-27). https://www.who.int/tools/flunet

9. Agence de la santé publique du Canada. Comité consultatif national de l'immunisation (CCNI). Chapitre sur la grippe du Guide canadien d'immunisation et Déclaration sur la vaccination antigrippale pour la saison 2020-2021. Ottawa (ON) : ASPC; 2020 (accédé 2021-07-28). https://www. canada.ca/fr/sante-publique/services/publications/vaccinsimmunisation/guide-canadien-immunisation-declarationvaccination-antigrippale-2020-2021.html
10. Agence de la santé publique du Canada. Grippe (influenza) : Surveillance. Surveillance de l'influenza : Du 23 mai 2021 au 19 juin 2021 (semaines de déclaration 21-24). Ottawa (ON) : ASPC; 2021 (accédé 2021-09-03). https://www.canada.ca/fr/ sante-publique/services/publications/maladies-et-affections/ surveillance-influenza/2020-2021/semaines-21-24-23-mai-19juin-2021.html

11. Agence de la santé publique du Canada. Bulletin des agents pathogènes des voies respiratoires émergents : Numéro 47, novembre 2020. Ottawa (ON) : ASPC; 2020 (accédé 2021-07-28). https://www.canada.ca/fr/sante-publique/ services/surveillance/bulletin-agents-pathogens-voiesrespiratoires-emergents/2020/novembere.html

12. Agence de la santé publique du Canada. Bulletin des agents pathogènes des voies respiratoires émergents : Numéro 52 , avril 2021. Ottawa (ON) : ASPC; 2020 (accédé 2021-07-28). https://www.canada.ca/fr/sante-publique/services/ surveillance/bulletin-agents-pathogens-voies-respiratoiresemergents/2021/avril.html

13. Agence de la santé publique du Canada. Bulletin des agents pathogènes des voies respiratoires émergents : Numéro 54, juin 2021. Ottawa (ON) : ASPC; 2020 (accédé 2021-07-28). https://www.canada.ca/fr/sante-publique/services/ surveillance/bulletin-agents-pathogens-voies-respiratoiresemergents/2021/juin.html

14. World Health Organization. Global Influenza Programme. Recommended composition of influenza virus vaccines for use in the 2020-2021 northern hemisphere influenza season. Geneva, Switzerland: WHO; 2020 (accédé 2021-07-28). https://www.who.int/publications/m/item/recommendedcomposition-of-influenza-virus-vaccines-for-use-in-the-20212022-northern-hemisphere-influenza-season

15. The Academy of Medical Sciences. COVID-19: Preparing for the future. London, UK: AMS; 2021(accédé 2021-07-30). https://acmedsci.ac.uk/file-download/4747802

16. Baker RE, Park SW, Yang W, Vecchi GA, Metcalf CJ, Grenfell BT. The impact of COVID-19 nonpharmaceutical interventions on the future dynamics of endemic infections. Proc Natl Acad Sci USA 2020;117(48):30547-53. DOI PubMed

17. Centers for Disease Control and Prevention. Selecting Viruses for the Seasonal Influenza Vaccine. Atlanta (GA): CDC; (modifié 2021-08-31; accédé 2021-07-30). https://www.cdc.gov/flu/prevent/vaccine-selection.htm 